\title{
Surgically induced degenerative changes in the femorotibial joints by total medial meniscectomy in minipigs closely resemble late-stage osteoarthritis
}

\author{
Won-Jae Lee', Byung-Joon Park ${ }^{1}$, Hyeon-Jeong Lee ${ }^{2}$, Si-Jung Jang ${ }^{2}$, Sung-Lim Lee ${ }^{2}$, Jae-Hoon Lee ${ }^{2}$, \\ Gyu-Jin Rho², Seung-Joon Kim ${ }^{1, *}$ \\ ${ }^{1}$ College of Veterinary Medicine, Kyungpook National University, Daegu 41566, Korea \\ ${ }^{2}$ College of Veterinary Medicine, Gyeongsang National University, Jinju 52828, Korea
}

\begin{abstract}
Animal models of osteoarthritis (OA) have played a key role in understanding the etiology of OA and in the development of new therapeutic strategies. Although pigs have an advantage as an animal disease model due to their similarity to humans, there are few studies on the induction of OA in minipigs. Therefore, this study aimed to characterize disease progression of OA in total medial meniscectomy (TMM)-operated skeletally mature minipigs, up to day 180 postoperatively. There were no significant alterations in vital signs or hematological indices throughout the observation period. However, clinical manifestations of OA in the medial femoral condyles of TMM-operated minipigs were progressive, depending on postoperative duration, with respect to osteophytes formation and roughened surfaces on radiological observation, cartilage erosion under macroscopic examination, and severe cartilage defects including fibrillation, vertical fissures, and cartilage denuding on histopathological observation, with the highest score indicating latestage OA on day 180 and without indicating apparent variation between subjects. In particular, the lateral femoral condyles were also degenerated, possibly due to localization of weight-bearing from both menisci to the lateral meniscus. Therefore, TMM in minipigs is suitable for reproducible induction of degenerative changes in the femorotibial joints that closely resemble late-stage OA, and is suitable for use in further research.
\end{abstract}

Keywords: osteoarthritis, minipig, meniscectomy, animal model

*Corresponding author

Seung-Joon Kim

College of Veterinary Medicine,

Kyungpook National University, 80

Daehak-ro, Buk-gu, Daegu 41566, Korea

Tel: $+82-53-950-5971$

Fax: +82-53-950-5994

E-mail: kjoon00@knu.ac.kr

ORCID:

Won-Jae Lee:

https://orcid.org/0000-0003-1462-7798

Byung-Joon Park:

https://orcid.org/0000-0003-1901-0869

Hyeon-Jeong Lee

https://orcid.org/0000-0002-2154-239X

Si-Jung Jang:

https://orcid.org/0000-0003-3829-6807

Sung-Lim Lee:

https://orcid.org/0000-0002-1055-8097

Jae-Hoon Lee:

https://orcid.org/0000-0002-8340-3694

Gyu-Jin Rho:

https://orcid.org/0000-0002-6264-0017

Seung-Joon Kim:

https://orcid.org/0000-0002-8521-8898

Conflict of Interest

The authors declare no conflicts of interest.

Received: Novemer 30, 2018

Revised : January 11, 2019

Accepted: February 8, 2019

\section{Introduction}

Osteoarthritis (OA) in humans has the highest prevalence among all forms of arthritis worldwide and may be defined as a complex disease involving erosion of cartilage and bone, osteophyte formation, synovitis, and joint capsule fibrosis [1-3]. The most common treatments for OA, such as analgesics, steroids, and pro-inflammatory cytokine inhibitors, have targeted attenuation of the symptoms; however, since these drugs have shown varying and limited therapeutic effects, there is a great unmet need for the development of new therapeutic strategies to completely decrease pain and effectively recover joint function [2-4].

Because OA models of small animals have merits, such as low cost, ease of handling, and availability of housing, they have been extensively used in OArelated researches. However, they have also presented critical disadvantages in terms of dissimilarities in joint structure with humans, as well as limitations of the translational potential due to physiological and genetic differences compared to humans; in addition, they cannot sufficiently mimic human diseases as animal models of OA $[2,5]$. Owing to these differences, the focus has shifted to OA models in large animals in recent years $[2,4,5]$. Among large animals, pigs are most similar to humans with respect to anatomy, neurobiology, immune system, cardiovascular system, gastrointestinal tract, omnivorous tendency, and genome. In particular, they are suitable as a mimic of the OA milieu of humans due to anatomical similarities in joint size, weight-bearing requirements, and cartilage thickness, as well as potentially having advantages of long-term study with continuous sample collection, large specimen volume, and application of tools originally developed for humans (arthroscopy, magnetic resonance imaging [MRI], and artificial articulation) [6]. 
However, induction of $\mathrm{OA}$ in pigs has been reported in only a few articles; in most cases, partial medial meniscectomy (PMM)- or anterior cruciate ligament transection (ACLT)operated juvenile pigs were involved in studies with short postoperative durations. Unfortunately, these reports were unable to fully elucidate disease progression in OA-induced pigs because of the use of skeletally immature juvenile pigs, a short postoperatively OA induction duration, lack of total medial meniscectomy (TMM)-induced OA model, and insufficient background data for general postoperative veterinary clinical observations [1, 3, 7-9].

Therefore, in the present study, we evaluated the changes in OA-related clinical manifestations related to behavioral observation, body temperature, pulse, respiration, blood pressure (BP) measurement, hematological assessment, radiological observation, and macroscopic/microscopic examinations, in TMM-operated skeletally mature minipigs throughout a long-term clinical observation of 180 days. The aim of the present study was to ensure the reproducibility of the surgically induced OA model in a large animal and to characterize the disease progression of OA in minipigs.

\section{Materials and Methods}

\section{Ethics and animals}

All procedures including TMM, animal care, and animal sacrifice were approved by the Institutional Animal Care and Use Committee (IACUC No. NP14060). A total of 15 healthy, 24-36-month-old, non-castrated male specific-pathogen-free minipigs (Prestige Bioresearch, Singapore) were used in the present study. Their average age was regarded as indicative of skeletal maturity in accordance with previous articles [1, $2,4,5]$. Environmental conditions similar to those in a previous study were provided to the minipigs [10].

\section{TMM and postoperative procedure}

The minipigs were randomly divided into 2 groups classified as TMM-operated $(\mathrm{n}=10)$ and sham-operated $(\mathrm{n}=5)$ minipigs and monitored on day 0 , day 90 , and day 180 . In brief, all animals were sedated with intramuscular injections of ketamine $(10 \mathrm{mg} / \mathrm{kg})$ and xylazine $(2.5 \mathrm{mg} / \mathrm{kg})$ with anesthesia maintained by isoflurane inhalation. A straight, $5 \mathrm{~cm}$ longitudinal cutaneous incision was made over the distal patella to the proximal tibial plateau of the hind leg. The medial side of the joint capsule was incised from the adductor tubercle of the femur to the anteromedial aspect of the tibia and then opened by lateral dislocation of the patella to expose the anterior femoral condyle and tibial plateau (Fig. 1A). After dissecting the medial collateral ligament, the anterior horn of the medial meniscus was clamped with forceps and completely separated using a blade along the tibial plateau (Fig. 1B). The same operation was repeated in another hind leg to induce OA in both legs in accordance with a previous report [8]. The sham surgery was conducted in the same manner until joint opening, except for performing TMM, to form a control group in agreement with the recommendation in a previous article [4]. The surgical site was closed in a routine manner and all animals were treated with antibiotics (Baytril; Bayer Healthcare, Germany) and analgesics (Overxicam; Over Medicina Veterinaria, Argentina) for 3 days in accordance with manufacturer's dosage. From 2 weeks after surgery, all minipigs were made to exercise for $2 \mathrm{~h}$ daily at an in-house playground, in agreement with the importance of exercise in a surgically induced OA model [7]. The minipigs were periodically monitored following the veterinary clinical observation schedules or sacrificed on day 90 (half of the TMM-operated minipigs, $\mathrm{n}=5$; TMM \#1) or day 180 (all sham-operated minipigs and remaining TMM-operated minipigs, each $\mathrm{n}=5$; sham and TMM \#2), as shown in Fig. 1C.

\section{In-life observation}

Behavioral observations were performed by 3 different caretakers and duration of playing, resting, and sleeping times were recorded in accordance with the definitions in a previous study [10]. The lameness of both knees of each group was scored by 3 different evaluators in accordance with numerical rating scales employed for lameness assessment in a dog model [11]. Body temperature, pulse, and res-
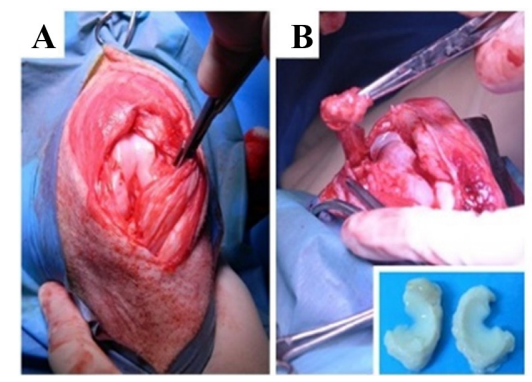

C
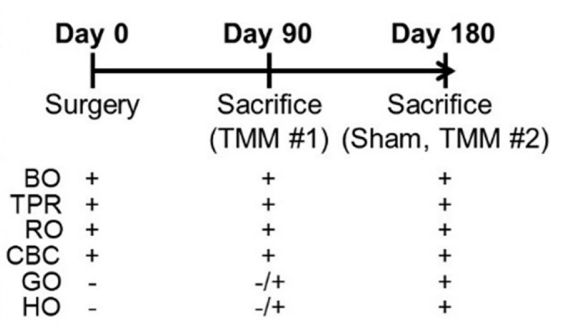

Fig. 1. TMM and postoperative procedure. (A) Exposure of the anterior femoral condyle and tibial plateau by the lateral dislocation of the patella. (B) TMM along the tibial plateau. (C) The schedule for monitoring BO, vital signs with TPR, as well as blood pressure measurement, RO, hematological observation for $\mathrm{CBC}$, macroscopic $\mathrm{GO}$ with measurement of the synovial fluid volume and microscopic HO. Sham, sham-operated minipigs; TMM \#1, TMM-operated minipigs on postoperative day 90; TMM \#2, TMM-operated minipigs on postoperative day 180; TMM, total medial meniscectomy; BO, behavioral observations; TPR, temperature, pulse, and respiration; $\mathrm{RO}$, radiological observation; $\mathrm{CBC}$, complete blood count; $\mathrm{GO}$, gross observation; HO, histopathological observation. 
piration (TPR), as well as BP measurement, were monitored by 3 different evaluators; body temperature $\left({ }^{\circ} \mathrm{C}\right.$ ) or BP (systolic arterial BP, diastolic arterial BP, and mean arterial BP; $\mathrm{mmHg}$ ) with heart rate (frequencies/min) or respiratory rate (frequencies/min) was measured using a digital centigrade thermometer or evaluated via a child-sized non-invasive BP cuff with the patient monitor (Dräger, Germany) at the hind limb of minipigs or counted when minipigs were comfortably resting in their own pen, respectively. Radiological images of the femorotibial joints were taken to observe the morphological changes using a C-arm X-ray system (Zen2090; Genoray, Korea); the ratio of degenerative femoral condyle (osteophyte formation and roughened surface) in both knees of each group (the number of degenerative femoral condyle/total number of femoral condyle) was assessed following consensual agreement of diagnosis from 2 different veterinarians. A small volume of blood was collected from the external jugular vein of each minipig and handled in a routine manner to assess complete blood count (CBC) using an ADVIA 2120 Hematology Analyzer (Siemens, USA).

\section{Macroscopic and microscopic examination}

The minipigs were euthanized (Fig. 1C) on day 90 (TMM \#1) or day 180 (sham and TMM \#2) with an overdose of thiopental sodium. The femorotibial joints were collected, and then the synovial fluid (SF) was carefully collected using a $10 \mathrm{~mL}$ syringe after gently opening the joint cavity [6]. For gross observation of the femorotibial joints, the femur and tibia were individually separated and macroscopically evaluated in accordance with the International Cartilage Repair Society (ICRS) macroscopic score [12]. For histopathological assessment, weight-bearing regions, where the cartilages were severely degraded, of both the medial and lateral femoral condyles were harvested (each size: $1 \mathrm{~cm} \times 1 \mathrm{~cm} \times 1 \mathrm{~cm}$ ), fixed with $10 \%$ formaldehyde (Sigma, USA) for 3 days, decalcified for $24 \mathrm{~h}$, dehydrated, embedded in paraffin, sectioned, and stained with Masson's trichrome (Sigma). The articular cartilage degeneration was quantitatively assessed by 3 different evaluators under a light microscope (Nikon, Japan), in accordance with the scoring system from the Osteoarthritis Research Society International (OARSI) [13].

A

Daily life

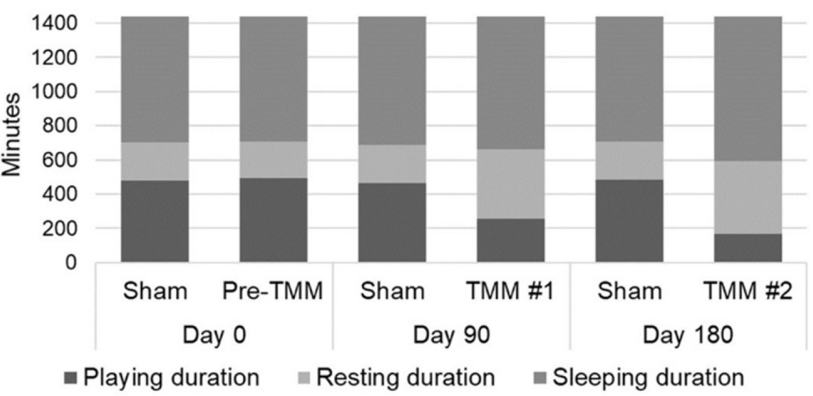

B

$$
\begin{array}{lll}
\text { Day } 0 & \text { Day } 90 & \text { Day } 180
\end{array}
$$

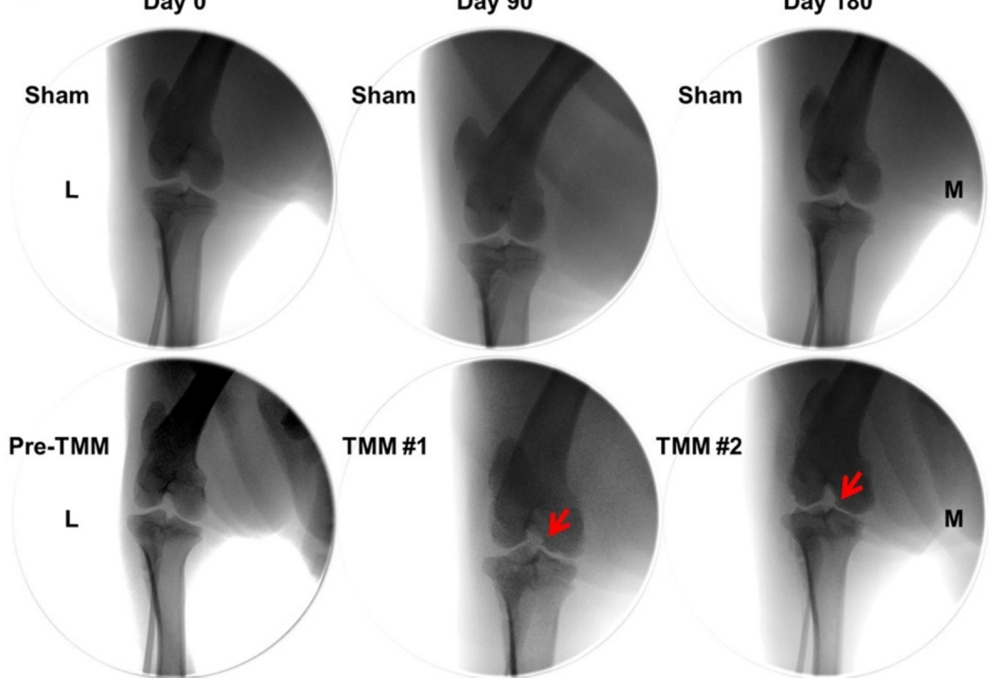

Fig. 2. In-life observations for daily life monitoring and radiological observation of sham- and TMM-operated minipigs. (A) Daily life investigation of behavioral tendencies including playing, resting, and sleeping duration. (B) Radiological observation of the femorotibial joint of sham- and TMM-operated minipigs, red arrows indicate osteophytes formation. Sham, sham-operated minipigs; TMM \#1, TMM-operated minipigs on postoperative day 90; TMM \#2, TMM-operated minipigs on postoperative day 180; TMM, total medial meniscectomy; pre-TMM, minipigs before total medial meniscectomy; L, lateral direction; M, medial direction. 


\section{Statistical analysis}

The data were analyzed using PASW 18 (SPSS, USA) software. Significant differences among groups were compared by using the Kruskal-Wallis test, followed by Bonferroni's correction. A $p$ value $<0.05$ was considered to be statistically significant. Data are presented as mean \pm SD values.

\section{Results}

\section{In-life observation}

Behavioral observations of daily life and lameness were postoperatively evaluated. With the passage of time in the postoperative period, TMM-operated minipigs were increasingly reluctant to move and play, in comparison with shamoperated minipigs (Fig. 2A). Moreover, lameness was significantly greater $(p<0.05)$ in TMM \#2 than in other groups (Table 1). Radiological observation showed the formation of osteophytes and presence of roughened surfaces in the medial femoral condyle in both TMM \#1 and \#2; progressive degradation of the femoral condyle was identified postoperatively and was duration dependent (Fig. 2B and Table 1). With regard to TPR, BP measurements, and hematological indices, the numerical values obtained from sham- and TMMoperated minipigs were within the normal reference range and not significantly different among groups throughout the experimental period (Table 1) [14, 15].

\section{Macroscopic examination}

While the cartilages of femorotibial specimens from shamoperated minipigs were intact and glossy without any clinical manifestation on gross observation, those obtained from TMM-operated minipigs demonstrated severe OA and were classified as grade $4(1.8 \pm 0.2$ in TMM \#1 and $0.5 \pm 0.5$ in TMM \#2); the specimens demonstrated cartilage defects with

Table 1. In-life observation of sham- and TMM-operated minipigs

\begin{tabular}{|c|c|c|c|c|c|c|}
\hline \multirow{2}{*}{ Variables } & \multicolumn{2}{|c|}{ Day 0} & \multicolumn{2}{|c|}{ Day 90} & \multicolumn{2}{|c|}{ Day 180} \\
\hline & Sham & Pre-TMM & Sham & TMM \#1 & Sham & TMM \#2 \\
\hline \multicolumn{7}{|l|}{ NRS for lameness } \\
\hline Score & $0.1 \pm 0.3$ & $0.1 \pm 0.3$ & $0.1 \pm 0.3$ & $0.5 \pm 0.5$ & $0.1 \pm 0.3$ & $2.5 \pm 0.7^{*}$ \\
\hline \multicolumn{7}{|c|}{ Radiological assessment $^{\dagger}$} \\
\hline Osteophyte & $0 / 10$ & $0 / 20$ & $0 / 10$ & $2 / 10$ & $0 / 10$ & $5 / 10$ \\
\hline Roughened surface & $0 / 10$ & $0 / 20$ & $0 / 10$ & $4 / 10$ & $0 / 10$ & $7 / 10$ \\
\hline \multicolumn{7}{|c|}{ Vital sign check and BP measurement } \\
\hline $\mathrm{BT}\left({ }^{\circ} \mathrm{C}\right)$ & $37.7 \pm 0.2$ & $37.6 \pm 0.6$ & $37.7 \pm 0.3$ & $37.3 \pm 0.5$ & $37.4 \pm 0.4$ & $37.4 \pm 0.3$ \\
\hline SAP $(\mathrm{mmHg})$ & $128.0 \pm 7.5$ & $130.5 \pm 10.3$ & $127.5 \pm 11.9$ & $128.3 \pm 11.1$ & $129.3 \pm 13.8$ & $125.8 \pm 7.9$ \\
\hline DAP (mmHg) & $58.3 \pm 7.1$ & $59.8 \pm 10.6$ & $61.8 \pm 6.8$ & $61.3 \pm 7.0$ & $60.3 \pm 7.9$ & $58.0 \pm 6.3$ \\
\hline MAP (mmHg) & $84.5 \pm 6.7$ & $85.8 \pm 4.3$ & $82.5 \pm 5.2$ & $83.8 \pm 6.2$ & $83.3 \pm 6.1$ & $86.0 \pm 4.7$ \\
\hline HR (freq./min) & $82.1 \pm 7.7$ & $84.0 \pm 6.3$ & $84.2 \pm 6.6$ & $82.4 \pm 6.0$ & $83.3 \pm 5.0$ & $87.5 \pm 7.1$ \\
\hline $\mathrm{RR}$ (freq./min) & $13.5 \pm 3.1$ & $13.0 \pm 3.9$ & $12.5 \pm 4.2$ & $13.8 \pm 2.9$ & $12.3 \pm 4.4$ & $13.5 \pm 3.7$ \\
\hline \multicolumn{7}{|c|}{ Hematological assessment (CBC) } \\
\hline WBC $\left(10^{3} / \mu \mathrm{L}\right)$ & $14.3 \pm 3.7$ & $14.2 \pm 4.1$ & $14.1 \pm 4.2$ & $14.0 \pm 4.2$ & $14.5 \pm 3.1$ & $11.1 \pm 3.6$ \\
\hline $\mathrm{RBC}\left(10^{3} / \mu \mathrm{L}\right)$ & $5.9 \pm 0.7$ & $6.1 \pm 1.0$ & $5.8 \pm 1.1$ & $6.4 \pm 0.7$ & $6.1 \pm 0.9$ & $6.0 \pm 0.6$ \\
\hline HGB (g/dL) & $12.2 \pm 1.5$ & $13.4 \pm 1.9$ & $12.0 \pm 1.6$ & $13.9 \pm 1.0$ & $12.4 \pm 3.1$ & $13.5 \pm 1.0$ \\
\hline HCT (\%) & $36.3 \pm 5.1$ & $37.8 \pm 3.1$ & $35.9 \pm 5.7$ & $38.1 \pm 3.7$ & $36.7 \pm 7.2$ & $39.8 \pm 2.8$ \\
\hline MCV (fL) & $60.5 \pm 3.7$ & $60.5 \pm 3.3$ & $58.7 \pm 5.0$ & $60.4 \pm 2.9$ & $59.5 \pm 3.4$ & $61.1 \pm 1.2$ \\
\hline MCH (pg) & $20.7 \pm 1.4$ & $20.6 \pm 1.7$ & $20.0 \pm 4.3$ & $20.8 \pm 1.0$ & $20.7 \pm 2.1$ & $20.9 \pm 0.3$ \\
\hline MCHC (g/dL) & $32.7 \pm 1.4$ & $33.3 \pm 2.5$ & $32.0 \pm 3.1$ & $34.2 \pm 0.7$ & $32.2 \pm 3.0$ & $33.0 \pm 0.3$ \\
\hline $\operatorname{PLT}\left(10^{3} / \mu \mathrm{L}\right)$ & $382 \pm 102$ & $366 \pm 85$ & $401 \pm 101$ & $383 \pm 66$ & $385 \pm 78$ & $398 \pm 32$ \\
\hline NEU $\left(10^{3} / \mu \mathrm{L}\right)$ & $6.6 \pm 3.0$ & $6.7 \pm 2.7$ & $6.7 \pm 2.9$ & $7.7 \pm 2.6$ & $6.4 \pm 2.1$ & $4.8 \pm 2.8$ \\
\hline LYM $\left(10^{3} / \mu \mathrm{L}\right)$ & $6.9 \pm 1.3$ & $6.3 \pm 1.9$ & $7.1 \pm 1.6$ & $6.6 \pm 1.7$ & $6.8 \pm 1.5$ & $5.1 \pm 1.0$ \\
\hline MONO $\left(10^{3} / \mu \mathrm{L}\right)$ & $0.8 \pm 0.1$ & $0.7 \pm 0.2$ & $0.7 \pm 0.2$ & $0.6 \pm 0.1$ & $0.9 \pm 0.2$ & $0.6 \pm 0.2$ \\
\hline $\operatorname{RET}\left(10^{9} / \mu \mathrm{L}\right)$ & $54.8 \pm 47.6$ & $73.4 \pm 50.5$ & $80.5 \pm 64.3$ & $54.1 \pm 15.4$ & $75.6 \pm 57.3$ & $30.1 \pm 3.7$ \\
\hline
\end{tabular}

Data are shown as mean \pm SD or number (\%).

TMM, total medial meniscectomy; NRS, numerical rating scale; BP, blood pressure; BT, body temperature; SAP, systolic arterial blood pressure; DAP, diastolic arterial blood pressure; MAP, mean arterial blood pressure; HR, heart rate; RR, respiratory rate; $\mathrm{CBC}$, complete cell count; WBC, white blood cell; RBC, red blood cell; HGB, hemoglobin; HCT, hematocrit; MCV, mean corpuscular volume; MCH, mean cell hemoglobin; MCHC, mean corpuscular hemoglobin concentration; PLT, platelet; NEU, neutrophil; LYM, lymphocyte; MONO, monocyte; RET, reticulocyte.

${ }^{*}$ Indicates a significant difference; ${ }^{\top}$ Number of degenerative femoral condyles/total number of femoral condyles. 
A
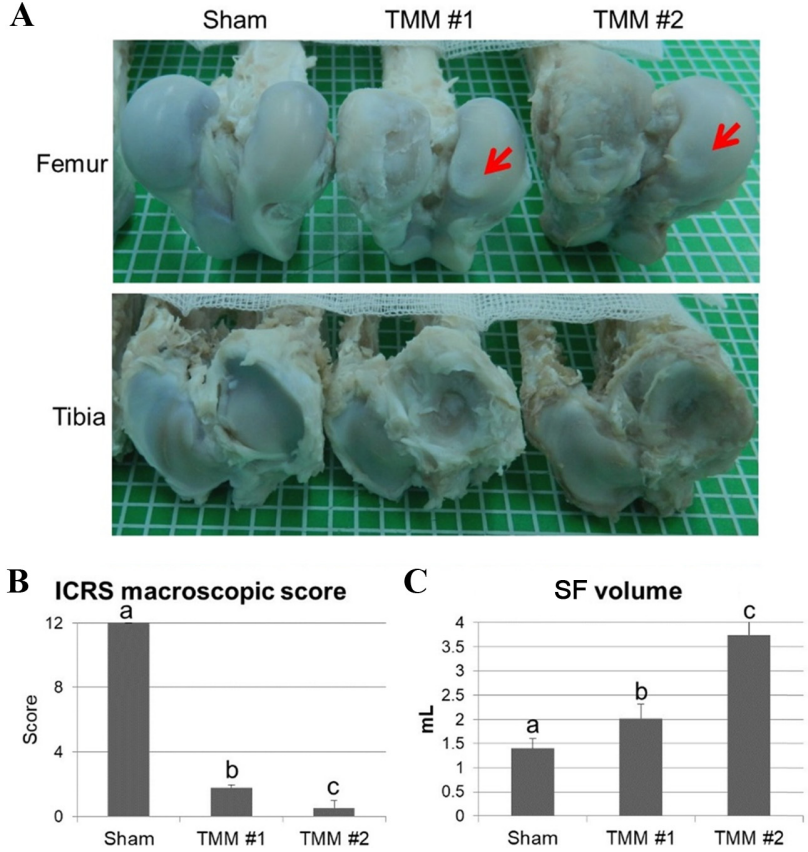

Fig. 3. Macroscopic examination of the femoral condyle and tibial plateau of sham- and TMM-operated minipigs. (A) Severe cartilage defects (erosion, roughened surface, and large fissures) in the medial femoral condyle and tibial plateau, and mild cartilage abrasion (red arrows) in the lateral femoral condyle. (B) ICRS macroscopic score from the collected femurs of shamand TMM-operated minipigs. (C) Measurement of the SF volume from sham- and TMM-operated minipigs. Graphs present mean \pm SD values. Sham, sham-operated minipigs; TMM \#1, TMM-operated minipigs on postoperative day 90; TMM \#2, TMM-operated minipigs on postoperative day 180; TMM, total medial meniscectomy; ICRS, International Cartilage Repair Society; SF, synovial fluid. ${ }^{\mathrm{a}, \mathrm{b}, \mathrm{c}}$ Indicated significant differences.

respect to cartilage erosion, cartilage irregularity, roughened cartilage surface, and large fissures in the medial femoral condyle and tibial plateau (Fig. 3A and B). Furthermore, the lateral femoral condyle in TMM-operated minipigs also showed mild cartilage damage with abrasion (Fig. 3A, red arrows). The elevation of SF volume, an index for OA [6], was significant $(p<0.05)$ in TMM-operated minipigs in comparison with that of sham-operated minipigs $(1.4 \pm 0.2 \mathrm{~mL})$; in addition, a postoperative duration-dependent elevation of SF volume identified between TMM \#1 $(2.0 \pm 0.3 \mathrm{~mL})$ and TMM \#2 $(3.7 \pm 0.5 \mathrm{~mL})$ was significant $(p<0.05)$ (Fig. $3 \mathrm{C}$ ).

\section{Microscopic examination}

Compared to the sham-operated minipigs, the histopathology of the femoral condyles after staining with Masson's trichrome demonstrated a severe progression of the clinical manifestation of $\mathrm{OA}$ in TMM-operated minipigs during the postoperative duration (Fig. 4). The histological images obtained from sham-operated minipigs presented an intact cartilage surface with normal structure and appropriate cell distribu-
A

Day 90

Day 180
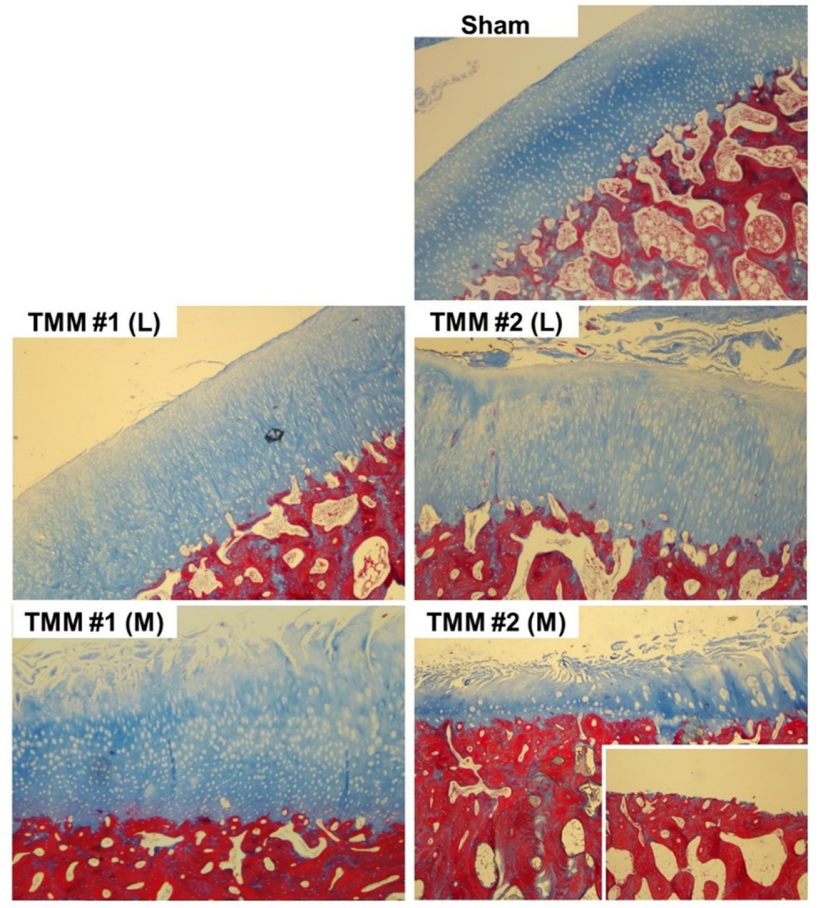

B

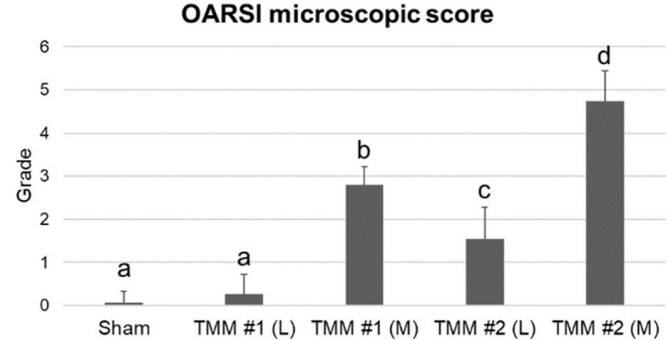

Fig. 4. Microscopic examination of the femoral condyle of sham- and TMM-operated minipigs. (A) Histopathology of femoral condyles after staining with Masson's trichrome. Large window in TMM \#2 (M) is a representative image of cartilage fibrillation, matrix discontinuity, vertical fissures into the mid zone, disorientation of chondron columns, and severe alteration of cellular distribution; small window in TMM \#2 (M) shows cartilage erosion and denuding. (B) Assessment of osteoarthritis-related cartilage histopathology from the specimens of shamand TMM-operated minipigs in accordance with OARSI scoring system. Graph presents mean $\pm \mathrm{SD}$ values (magnification is $\times 40$ ). Sham, sham-operated minipigs; TMM \#1 (L) or TMM \#1 (M), the lateral or medial femoral condyles of TMM \#1, respectively; TMM \#2 (L) or TMM \#2 (M), the lateral or medial femoral condyles of TMM \#2, respectively; TMM, total medial meniscectomy; OARSI, Osteoarthritis Research Society International; $\mathrm{L}$, lateral direction; $\mathrm{M}$, medial direction. ${ }^{\mathrm{a}, \mathrm{b}, \mathrm{c}}$ Indicate significant differences.

tion, indicating grade 0 cartilage $(0.1 \pm 0.1)$ (sham in Fig. $4 \mathrm{~A})$. However, the medial femoral condyles of TMM \#1 (TMM \#1 [medial direction; M] in Fig. 4A) exhibited cartilage grade $2-3(2.8 \pm 0.4)$-related histological alterations in 
accordance with the OARSI scoring system; the TMM \#1 specimens demonstrated cartilage fibrillation, matrix discontinuity, vertical fissures into the mid zone, disorientation of chondron columns, and severe alteration of cellular distribution. There was further progressive degeneration in the cartilages of the medial femoral condyles in TMM \#2 (TMM \#2 [M] in Fig. 4A); the degeneration was classified as grade 4-5 $(4.7 \pm 0.7)$ and was more severe than the aforementioned alterations in TMM \#1 (TMM \#2 [M] in Fig. 4A), as well, there was cartilage erosion and denuding present (small window in TMM \#2 [M] in Fig. 4A). Overall, quantitative assessment in accordance with OARSI scoring system demonstrated that the postoperative duration-dependent cartilage degeneration in the medial femoral condyle of TMM-operated minipigs was significant $(p<0.05)$ among the sham, TMM \#1 and TMM \#2 groups (Fig. 4B). Furthermore, in agreement with macroscopic examinations performed in the present study, the cartilages of the lateral femoral condyles of TMM \#2 (TMM \#2 [lateral direction; L] in Fig. 4A) were mildly degraded, presented superficial fibrillation and abrasion, and were assessed as grade $1-2(1.5 \pm 0.7)$; the TMM \#2 (L) OA grade value was significantly $(p<0.05)$ higher than those from the sham group and that for the lateral femoral condyles of TMM \#1 (0.3 \pm 0.4 ; TMM \#1 [L] in Fig. 4A).

\section{Discussion}

There have been great efforts undertaken to understand the etiology of human OA. Similarly, extensive trials have been conducted to develop effective therapeutic strategies $[1,2,4]$. Since OA studies are difficult to be directly conducted in humans, animal models of OA have been widely employed because of several benefits such as estimating the exact time of disease onset, controlling environmental influences and causes, consistent OA phenotype, development of new genetic models, and permitting aggressive harvesting of tissue samples; therefore, establishment of a reproducible induction method of OA in animals and characterization of its disease progression is important [2, 3].

Although the pig has several advantages as an animal disease model such as anatomically similar joints with human, large specimen volume, feasibility of long-term studies with continuous sampling, trainability, provision of more clinically relevant data, and direct application of medical devices originally developed for humans, only a few articles have reported OA models in pigs [2, 3, 5]. In brief, when 15-weekold ACLT-operated minipigs with or without application of bioactive scaffolds were observed for 12 weeks, ACLT-operated minipigs with scaffold application demonstrated improvements in structural properties and potential to repair cartilage damage [16]. The application of mesenchymal stem cells in PMM-operated juvenile minipigs demonstrated regeneration of the resected meniscus and protection of the medial femoral cartilage after 16 weeks of the operation [8]. The 9-weekold PMM-operated minipigs were monitored for 20 days and compared with sham-operated minipigs; whereas the PMMoperated minipigs demonstrated early-stage OA, the shamoperated minipigs did not present any pathological changes [7]. However, these aforementioned reports have several limitations to fully elucidating disease progression in $\mathrm{OA}-$ induced pigs including the usage of skeletally immature juvenile pigs, a short postoperatively OA induction duration, the lack of a TMM-induced OA model, and insufficient background data for general postoperative veterinary clinical observations [1-5, 17]. To overcome these limitations, we induced OA by TMM to skeletally mature minipigs and monitored the clinical manifestations for a duration of 180 days. The sham-operated minipigs in the present study were unaffected by surgery and demonstrated glossy cartilage classified as grade 1 (normal) on macroscopic examination or intact cartilage classified as grade 0 (normal) on microscopic examination following the ICRS or OARSI scoring system, respectively (Figs. 3 and 4). In contrast, the femorotibial joints in TMM \#1 and TMM \#2 minipigs demonstrated severe abnormality $(1.8 \pm 0.2$ and $0.5 \pm 0.5)$ under macroscopic examination (Fig. 3A and B). In addition, the SF volume progressively elevated and was dependent on postoperative duration (Fig. 3C); moreover, it was increased in osteoarthritic and ACL-injured human patients by activation of synovium-related cells (synovial fibroblasts and macrophages) which produce SF under inflammatory milieu [6, 18]. While the femoral condyles of PMM-operated minipigs on day 20 microscopically exhibited mild OA symptoms, those from TMM-operated minipigs in the present study showed more progressive cartilage degradations depending on the postoperative duration with scores of $2.8 \pm 0.4$ in TMM \#1 and 4.7 \pm 0.7 in TMM \#2 (Fig. 4) [7]. In particular, the TMM \#2 specimens extensively presented late-stage clinical manifestations of $\mathrm{OA}$ in terms of cartilage fibrillation, matrix discontinuity, vertical fissures into the deep zone, and erosion and denuding of cartilage. In addition, the level of mild degraded cartilage of the lateral femoral condyle in TMM \#2 was significant $(p<0.05)$ with a microscopic score of $1.5 \pm 0.7$ in the present study; it was possible that OA progression in the lateral femoral condyle was caused by localization of weightbearing from both medial and lateral menisci to a lateral meniscus, in agreement with previous results for a sham group [19].

Among several OA induction methods, surgically induced OA by ACLT, PMM, and TMM are commonly performed in large animals to utilize rapid induction with easy reproducibility and to overcome the limitations derived from other induction methods such as insufficient mimicking of OA progress with intra-articular injection of certain components, variable disease incidence in spontaneous OA models, and absence of genetically modified OA models [3]. However, the selection of appropriate surgical induction methods based on the anatomical differences between species should be considered for the successful induction of OA in large animals. Whereas TMM in rabbits resulted in weak OA due to weight 
load in the lateral compartment of joints, TMM in dogs was feasible for the development of severe OA symptoms [17, 20]. In addition, ACLT in dogs or in sheep could result in cartilage erosion within months or little detectable cartilage damage even after 18 months, respectively [21, 22]. In case of the pig, ACLT might not always be useful for reproducible induction of $\mathrm{OA}$ because there was variable degradation of the cartilage between each ACLT-operated pig, ranging from smooth hyaline cartilage layer (normal) results to destroyed cartilage; moreover, correlation between disease progression of OA and detection of the degenerative cartilage by MRI was not identified [19]. In addition, ACLT-operated 6-monthold pigs exhibited mild degradation of the cartilage in the femoral condyle during macroscopic observation even at 6 weeks post-induction [9]. In the present study, all of the TMM-operated minipigs demonstrated severe arthritic symptoms in close accord with the highest grade in the ICRS and OARSI scoring system, without showing apparent variation between subjects (Figs. 3 and 4). Overall, it could be concluded that TMM in minipigs was anatomically and reproducibly suitable to induce severe OA, because the TMM produced direct mechanical impact between the femur condyle and tibial plateau, and the loads for weight-bearing were transferred through the medial compartment of the femorotibial joint.

We performed not only macroscopic and microscopic examinations but also in-life observations in the present study (Fig. 2 and Table 1). Whereas sham and TMM \#1 pigs exhibited a normal gait, TMM \#2 pigs presented the mild to moderate lameness. The TPR and BP measurements during the postoperative duration of TMM in the present study were within the normal range $[14,15]$. In the case of the OA model of dogs by medial patellar luxation (MPL), radiological alterations could be detectable only in the late-stage of OA, not in the early-stage [17]. In the present study, osteophytes formation and roughened surface of the medial femoral condyle were observed in TMM-operated minipigs on radiological examination (Fig. 2B); these results indicate that the effect of TMM in minipigs was sufficient to induce severe and late-stage OA. Currently, studies on CBC parameters of OA models in large animals, especially pigs, are limited. MPL-operated dogs exhibited significantly $(p<0.05)$ mild elevations in the number of platelets and hematocrit levels, but no considerable changes were observed in white blood cell (WBC)-related parameters [17]. As the naturally occurring OA, hip dysplasia in dogs resulted in an increase in the number of WBCs, but this was not clinically important because the numerical value was within the normal range [23]. The present study demonstrated no significant alteration of $\mathrm{CBC}$ parameters throughout the postoperative duration of TMM. Although WBC-related parameters in TMM \#2 presented decreased tendencies, compared to those of sham-operated minipigs and TMM \#1, they were not significant and remained within the normal range $[14,15]$.

Since no single animal model can perfectly mimic all aspects of the arthritic milieu in human OA, the characterization of various OA models using different species and induction methods is important for better understanding of the etiology of OA and effectively development of new therapeutic strategies. In this sense, the present study results indicate that TMM in skeletally mature minipigs could successfully and reproducibly induce degenerative changes in cartilage on day 180 that are representative of late-stage OA. We believe that the present results may contribute to further OA-related studies in large animals and be helpful in the choice of animal model, planning the experimental design for the timeline of OA progression, and standardization of monitoring methods.

\section{Acknowledgments}

This research was supported by Kyungpook National University Research Fund, 2017, and was conducted with Prestige BioResearch (Singapore 638117).

\section{References}

1. Cohen-Solal M, Funck-Brentano T, Hay E. Animal models of osteoarthritis for the understanding of the bone contribution. Bonekey Rep 2013;2:422.

2. Gregory MH, Capito N, Kuroki K, Stoker AM, Cook JL, Sherman SL. A review of translational animal models for knee osteoarthritis. Arthritis 2012;2012:764621.

3. Kuyinu EL, Narayanan G, Nair LS, Laurencin CT. Animal models of osteoarthritis: classification, update, and measurement of outcomes. J Orthop Surg 2016;11:19.

4. Teeple E, Jay GD, Elsaid KA, Fleming BC. Animal models of osteoarthritis: challenges of model selection and analysis. AAPS J 2013;15:438-446.

5. McCoy AM. Animal models of osteoarthritis: comparisons and key considerations. Vet Pathol 2015;52:803-818.

6. Sekiya I, Ojima M, Suzuki S, Yamaga M, Horie M, Koga H, Tsuji K, Miyaguchi K, Ogishima S, Tanaka H, Muneta T. Human mesenchymal stem cells in synovial fluid increase in the knee with degenerated cartilage and osteoarthritis. J Orthop Res 2012;30:943-949.

7. Cruz R, Ramírez C, Rojas OI, Casas-Mejía O, Kouri JB, Vega-López MA. Menisectomized miniature Vietnamese pigs develop articular cartilage pathology resembling osteoarthritis. Pathol Res Pract 2015;211:829-838.

8. Hatsushika D, Muneta T, Nakamura T, Horie M, Koga H, Nakagawa Y, Tsuji K, Hishikawa S, Kobayashi E, Sekiya I. Repetitive allogeneic intraarticular injections of synovial mesenchymal stem cells promote meniscus regeneration in a porcine massive meniscus defect model. Osteoarthritis Cartilage 2014;22:941-950.

9. Wei B, Zong M, Yan C, Mao F, Guo Y, Yao Q, Xu Y, Wang L. Use of quantitative MRI for the detection of progressive cartilage degeneration in a mini-pig model of osteoarthritis caused by anterior cruciate ligament transection. J Magn Reson Imaging 2015;42:1032-1038.

10. Jeon RH, Kim SJ, Lee WJ. The effect of preferable enrichments in the laboratory minipigs. J Embryo Transf 
24 Won-Jae Lee, Byung-Joon Park, Hyeon-Jeong Lee, Si-Jung Jang, Sung-Lim Lee, Jae-Hoon Lee, Gyu-Jin Rho, Seung-Joon Kim

2017;32:305-310

11. Impellizeri JA, Tetrick MA, Muir P. Effect of weight reduction on clinical signs of lameness in dogs with hip osteoarthritis. J Am Vet Med Assoc 2000;216:1089-1091.

12. van den Borne MP, Raijmakers NJ, Vanlauwe J, Victor J, de Jong SN, Bellemans J, Saris DB; International Cartilage Repair Society. International Cartilage Repair Society (ICRS) and Oswestry macroscopic cartilage evaluation scores validated for use in Autologous Chondrocyte Implantation (ACI) and microfracture. Osteoarthritis Cartilage 2007;15:1397-1402.

13. Pritzker KP, Gay S, Jimenez SA, Ostergaard K, Pelletier JP, Revell PA, Salter D, van den Berg WB. Osteoarthritis cartilage histopathology: grading and staging. Osteoarthritis Cartilage 2006;14:13-29.

14. Latimer KS, Mahaffey EA, Prasse KW. Duncan and Prasse's Veterinary Laboratory Medicine: Clinical Pathology. 4th ed. pp. 331-338, Wiley-Blackwell, New Jersey, 2003.

15. Plumb DC. Plumb's Veterinary Drug Handbook. 5th ed. pp. 1241-1249, Wiley-Blackwell, New Jersey, 2005.

16. Murray MM, Fleming BC. Use of a bioactive scaffold to stimulate anterior cruciate ligament healing also minimizes posttraumatic osteoarthritis after surgery. Am J Sports Med 2013;41:1762-1770.

17. Alam MR, Lee HB, Park SY, Lee YH, Kim IS, Kang HS, Kim NS. Changes in the haematobiochemical parameters in experimental stifle osteoarthritis in dogs. Pak J Biol Sci 2006;9:2869-2874.

18. Scanzello CR, Goldring SR. The role of synovitis in osteoarthritis pathogenesis. Bone 2012;51:249-257.

19. Reisig G, Kreinest M, Richter W, Wagner-Ecker M, Dinter D, Attenberger U, Schneider-Wald B, Fickert S, Schwarz ML. Osteoarthritis in the knee joints of Göttingen minipigs after resection of the anterior cruciate ligament? Missing correlation of MRI, gene and protein expression with histological scoring. PLoS One 2016;11:e165897.

20. Berjon JJ, Munuera L, Calvo M. Degenerative lesions in the articular cartilage after meniscectomy: preliminary experimental study in dogs. J Trauma 1991;31:342-350.

21. Brandt KD, Myers SL, Burr D, Albrecht M. Osteoarthritic changes in canine articular cartilage, subchondral bone, and synovium fifty-four months after transection of the anterior cruciate ligament. Arthritis Rheum 1991;34:1560-1570.

22. Cesana M, Appleyard RC, Jones HP, Murrell GAC. The effect of chronic ACL deficiency on the secondary restraints of the knee: a biomechanical study in a sheep model. Trans Annu Meet Orthop Res Soc 2003;28:803.

23. Nganvongpanit K, Itthiarbha A, Ong-Chai S, Kongtawelert P. Evaluation of serum chondroitin sulfate and hyaluronan: biomarkers for osteoarthritis in canine hip dysplasia. J Vet Sci 2008;9:317-325. 